\title{
SABERES POPULARES SOBRE Hydromedusa maximiliani (MIKAN, 1820) (TESTUDINES, CHELIDAE): UMA ABORDAGEM NO ENTORNO DE UMA UNIDADE DE CONSERVAÇÃO URBANA EM MINAS GERAIS, BRASIL
}

\author{
POPULAR KNOWLEDGE ABOUT Hydromedusa maximiliani (MIKAN, 1820) \\ (TESTUDINES, CHELIDAE): AN APPROACH AROUND AN URBAN \\ CONSERVATION UNIT IN MINAS GERAIS, BRAZIL
}

\begin{abstract}
Alessandra Rezende PEREIRA ${ }^{1}$, Thiago da Silva NOVATO ${ }^{2 *}$, Robson Henrique de CARVALHO ${ }^{1}$, Iara Alves NOVELLI ${ }^{1}$, Bernadete Maria de SOUSA ${ }^{1}$

${ }^{1}$ Laboratório de Répteis, Departamento de Zoologia, Instituto de Ciências Biológicas, Universidade Federal de Juiz de Fora; ${ }^{2}$ MirmecoLab, Departamento de Zoologia, Instituto de Ciências Biológicas, Universidade Federal de Juiz de Fora; autor para correspondência * thiago.novato@icb.ufjf.br

Submitted: 11/06/2019; Accepted: 19/08/2019
\end{abstract}

RESUMO: Hydromedusa maximiliani (Mikan, 1820) (Testudines, Chelidae) é uma espécie de Testudine de água doce endêmica da Mata Atlântica brasileira. Conhecido como cágado-pescoço-decobra, pode ser encontrado nos cursos d'água da Reserva Biológica Municipal Santa Cândida (RBMSC), uma área urbana protegida, que auxilia na qualidade ambiental de Juiz de Fora, MG. Como a relação de populações humanas com esta espécie de tartaruga ainda é pouco investigada, esta pesquisa objetivou realizar uma análise dos saberes populares sobre $H$. maximiliani no entorno da RBMSC. Foram realizadas 22 entrevistas semiestruturadas com moradores do entorno da RBMSC, constatandose que 13 moradores conhecem a espécie, suas características e sua biologia, porém a maioria demonstra desconhecer os usos alimentares e medicinais de espécies de cágados. Também foi possível registrar uma relação conflituosa da população com a Reserva pela retirada de recursos, como bambu e madeira, e ocupação do território. Assim, conclui-se que a relação da população local com o cágado era mais intrínseca ao passado e que o conhecimento e uso dessa espécie vêm sendo perdidos ao longo das gerações, principalmente devido ao avanço da urbanização. Assim, estratégias de conservação que incluam os moradores do entorno da RBMSC são fundamentais para sua proteção.

PALAVRAS-CHAVE: Áreas Protegidas, conflitos socioambientais, etnobiologia, répteis, tartaruga de água-doce.

ABSTRACT: Hydromedusa maximiliani (Mikan, 1820) (Testudines, Chelidae) is a species of freshwater testudine endemic to the Brazilian Atlantic Forest. Known as the snake-necked turtle, it can be found in the water courses of the Santa Cândida Municipal Biological Reserve (RBMSC), a protected urban area, which helps in the environmental quality of Juiz de Fora, MG. As the relationship of human populations with this species of turtle is still poorly investigated, this research aimed to carry out an analysis of popular knowledge about $H$. maximiliani in the surroundings of the RBMSC. Twenty-two semi-structured interviews were carried out with residents from the surroundings of the RBMSC. Of the total respondents, 13 residents know the species, its characteristics and its biology, but most of them are unaware of its use as food and medicine. It was also possible to register a conflicting relationship between the residents and the Reserve due to the withdrawal of resources, such as bamboo and wood, and occupation of the territory. Thus, we concluded that the relationship of the local population with the turtle was more intrinsic to the past and that the knowledge and use of this species has been lost over the generations, mainly due to the advance of urbanization. In this regard, conservation strategies that include the residents of the surroundings of the RBMSC are fundamental for their protection.

KEYWORDS: protected areas, socio-environmental conflicts, ethnobiology, reptiles, freshwater turtle 


\section{INTRODUÇÃO}

Hydromedusa maximiliani (Mikan, 1820) (Testudines, Chelidae), conhecida por cágado-da-serra ou cágado-pescoço-de-cobra, é uma espécie de tartaruga de água doce, endêmica da Mata Atlântica (SOUZA, 2005) e que habita córregos de água limpa e fria, sendo essa uma importante condição para a manutenção de suas populações (ICMBIO-RAN, 2011; NOVELLI et al., 2013; COSTA et al., 2015). A espécie está categorizada como "vulnerável" pela União Internacional para a Conservação da Natureza (IUCN, 2016) e com dados insuficientes pelo Livro Vermelho da Fauna Brasileira Ameaçada de Extinção (ICMBIO, 2018). Contudo, corre risco de extinção devido às ameaças à biodiversidade, principalmente pela perda e fragmentação de habitats e poluição (ROCHA et al., 2009; VOGT et al., 2015a).

Esta espécie foi registrada em 48 localidades brasileiras, distribuídas nos Estados da Bahia, Espírito Santo, Rio de Janeiro, São Paulo e Minas Gerais (COSTA et al., 2015). Chagas e Raposo-Filho (1999) registraram uma população de $H$. maximiliani no município de Juiz de Fora, Minas Gerais, na Reserva Biológica Municipal Santa Cândida (RBMSC) e desde o ano de 2000 estudos sobre a biologia, comportamento e ecologia da espécie vêm sendo realizados (NOVELLI e SOUSA, 2008; NOVELLI e SOUSA, 2007; NOVELLI et al., 2009a; NOVELLI et al., 2009b; NOVELLI et al., 2013; NOVELLI et al., 2014). Trata-se de uma pequena Unidade de Conservação Urbana criada pelo Decreto Municipal ${ }^{\circ}$ 2.904 de 03 de junho de 1983, localizada em área de conflito com loteamentos vizinhos (PJF, 2018).

Segundo Soanes et al. (2019), pequenas Áreas Protegidas (APs) urbanas podem apoiar e sustentar populações de espécies endêmicas. Adicionalmente, podem apresentar-se como provedoras de qualidade ambiental e benéficas aos seres humanos, interferindo positivamente na qualidade de vida e mitigando aspectos negativos da urbanização (SHANAHAN et al., 2015). Porém, são mais vulneráveis que as APs rurais, devido aos impactos do processo de urbanização, tais como a redução de habitat, introdução de espécies exóticas e contaminação de mananciais, que levam à perda de biodiversidade e promovem a redução dos serviços ecossistêmicos (MENEZES, 2005; GUIMARÃES e PELLIN, 2015). Ressalta-se que a criação e gestão de APs, de modo geral, podem gerar conflitos socioambientais com as comunidades locais (SOLIKU e SCHRAML, 2018), considerados positivos ou negativos para a proteção da biodiversidade (YOUNG et al., 2010).

Nesse contexto, torna-se um desafio minimizar os impactos causados pelo processo de urbanização na proteção dos recursos naturais. Para isso, é fundamental entender o contexto multifatorial das interações entre seres humanos e natureza, utilizando a Etnobiologia como ferramenta de pesquisa (ALVES; ALBUQUERQUE, 2018; ALVES, 2012). Ela pode auxiliar na avaliação dos impactos que as populações humanas causam sobre espécies nativas e no desenvolvimento de planos estratégicos para a conservação da biodiversidade (ALVES, 2012). Além disso, pode contribuir em propostas de gerenciamento de APs, influenciando no beneficiamento de populações humanas que vivem próximos a essas áreas, bem como na forma de se relacionar com os atributos da biodiversidade que compõem a paisagem em que se inserem (RAFAEL, 2017).

Considerando que a conservação da natureza possui uma íntima relação com questões sociais, econômicas, culturais e biológicas (SANDRONI e CARNEIRO, 2016), a relação dos quelônios de água doce da espécie $H$. maximiliani e a população humana no Brasil ainda é muito pouco investigada (NOVELLI e SOUSA, 2004). Assim, a partir da apresentação de registros que revelam aspectos importantes em relação à conservação de quelônios, o presente estudo realiza uma análise das interrelações estabelecidas entre pessoas e o cágado pescoço-de-cobra no entorno da RBMSC, Juiz de Fora, Minas Gerais, como estratégia de conservação desta espécie.

\section{MATERIAL E MÉTODOS \\ Área de estudo}

A pesquisa foi desenvolvida com a população do entorno da RBMSC, que possui uma área de 113,3 hectares, inserida no domínio fitogeográfico Mata Atlântica. Localiza-se a oeste do centro da cidade de

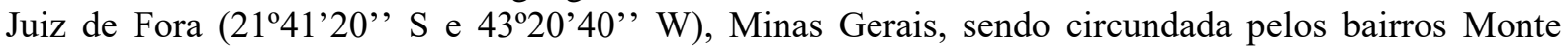
Castelo, Carlos Chagas, Milho Branco, Caiçaras e São Pedro (Figura 1).

A RBMSC é considera como uma área de zona especial pela Lei Municipal no 6.910 de 31 de maio de 1986, que está sujeita a um regime urbanístico mais restritivo e que estabelece as limitações urbanas com intuito de preservar os recursos naturais e realizar a proteção ambiental e ecológica (JUIZ DE FORA-MG, 1986). 


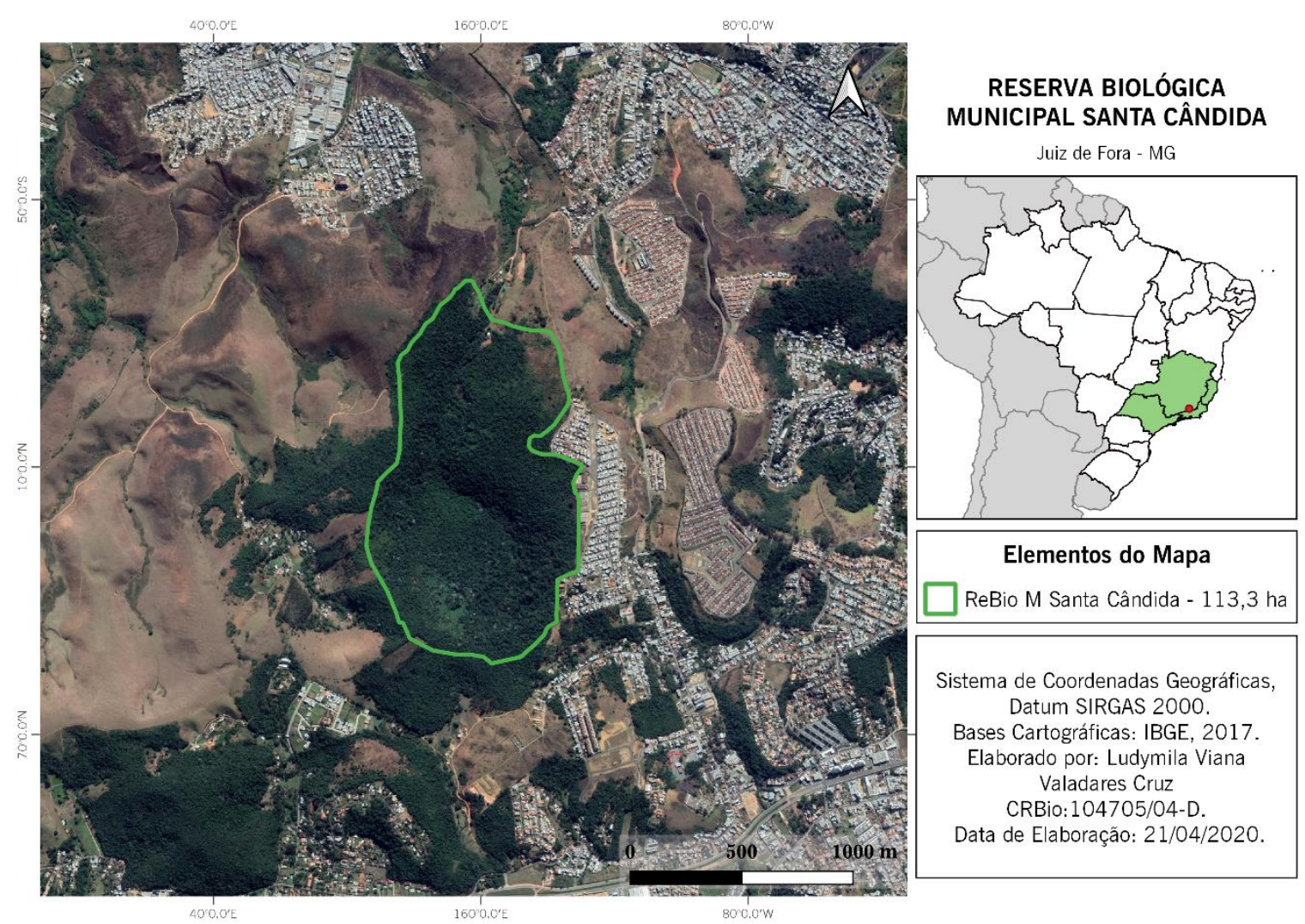

Figura 1: Localização da Reserva Biológica Municipal Santa Cândida (RBMSC), circundada pelos bairros Monte Castelo, Carlos Chagas, Milho Branco, Caiçaras e São Pedro.

\section{Coleta e análise dos dados}

Antes da realização das entrevistas, a proposta da pesquisa foi apresentada aos informantes que, quando interessados, assinaram o Termo de Consentimento Livre e Esclarecido, concordando em participar do trabalho. A coleta dos dados também foi autorizada pelo Comitê de Ética em Pesquisas da Universidade Federal de Juiz de Fora (CEP/UFJF), através do Parecer $\mathrm{N}^{\circ} 1.840 .829$ de 28 de novembro de 2016.

As informações relacionadas ao conhecimento local e interação entre as pessoas e o cágado foram coletadas através de entrevistas semiestruturadas (ALBUQUERQUE et al., 2019), contendo perguntas abertas e fechadas, durante os meses de maio e junho de 2017. Os informantes da pesquisa foram selecionados pelo método bola de neve (BAILEY, 1982), onde o primeiro entrevistado foi sugerido por uma pesquisadora que já tinha contato com a população local, com o objetivo de identificar os moradores locais que conhecem a espécie $H$. maximiliani. Uma amostragem aleatória simples também foi aplicada em dez casas da rua de acesso à RBMSC, onde se buscou entrevistar um morador de cada casa, sendo possível realizar nove destas dez entrevistas.

As entrevistas foram divididas em duas etapas: a primeira (pré-entrevista) consistiu em uma conversa prévia com o informante para levantar informações sobre a relação com a reserva, o conhecimento do cágado e o tempo em que residia na área. Após essa etapa, caso o entrevistado expressasse conhecer a reserva e o cágado, um formulário semiestruturado era aplicado contendo perguntas sobre conceito êmico, habitat, dieta, reprodução, uso para fins médicos e alimentares e a importância de conservação da RBMSC (Tabela 1).

Junto ao formulário, foi empregada uma prancha de identificação com fotos de quatro espécies de quelônios (Figura 2), como ferramenta de estímulo visual, onde os informantes puderam mostrar na prancha quais animais ocorriam na Reserva, sendo possível indicar mais de um animal.

A análise de dados foi realizada de forma qualitativa a partir de uma categorização das respostas, com a finalidade de construir um discurso coletivo que continha a distribuição das informações fornecidas. As informações do estímulo visual foram listadas em uma planilha digital e suas frequências calculadas. 

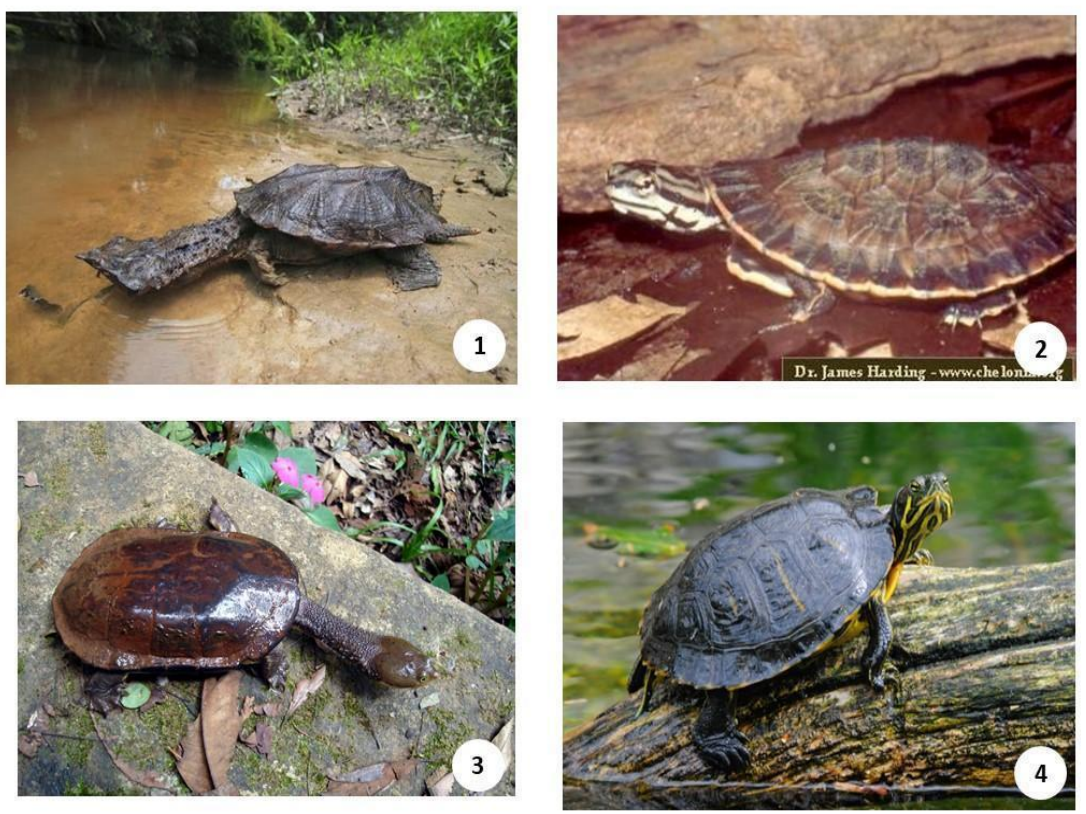

Figura 2: Prancha fotográfica utilizada como estímulo visual nas entrevistas. O número 1 corresponde à espécie mata-matá, Chelus fimbriatus (Fonte: Strange Animals). O número 2 refere-se ao cágado-de-barbicha, Phrynops geoffroanus (Fonte: World Chelonian Trust). O número 3, ao cágado pescoço-de-cobra, Hydromedusa maximiliani (Fonte: Novelli. I. A). E o número 4, ao cágado tigre-d'água, Trachemys scripta (Fonte: CalPhotos).

Tabela 1: Questões realizadas na entrevista semiestruturada.

\begin{tabular}{|l|l|}
\hline Q 1 & $\begin{array}{l}\text { Qual o tipo de contato o (a) Sr. (a) tem com a Reserva? ( ) lazer e diversão; ( ) coleta de recursos; ( ) } \\
\text { outros; ( ) não tenho contato. }\end{array}$ \\
\hline Q 2 & O (a) Sr. (a) já viu algum cágado ou tartaruga aqui na região? ( ) sim; ( ) não. \\
\hline Q 3 & Quais desses animais (mostra a prancha) o (a) Sr. (a) já viu aqui? ( ) 1; ( ) 2; ( ) 3; ( ) 4. \\
Q 4 & Como o (a) Sr. (a) chama esse animal? \\
\hline Q 5 & O que o (a) Sr. (a) acha que ele come? \\
Q 6 & E em que lugar ele vive? \\
Q 7 & $\begin{array}{l}\text { Como o (a) Sr. (a) acha que esse animal se reproduz? (resposta). Existem machos e fêmeas? } \\
\text { (resposta). Eles são diferentes? }\end{array}$ \\
Q 8 & O (a) Sr. (a) já viu alguém comendo alguma parte desse animal? (resposta). O (a) Sr. (a) já comeu? \\
Q 9 & $\begin{array}{l}\text { O (a) Sr. (a) sabe de alguém que usa esse animal ou alguma parte dele para tratar doenças? } \\
\text { Q 10 sim; ( ) não. Quais doenças? }\end{array}$ \\
\hline Q 11 (a) Sr. (a) acha que em 10 anos a quantidade de tartaruga aumentou ou diminuiu na Reserva? \\
Q 12 & O (a) Sr. (a) acha que esse animal sofre alguma ameaça? ( ) sim; ( ) não. Qual?
\end{tabular}

\section{RESULTADOS E DISCUSSÃO}

Foi realizado um total de 22 entrevistas com a população do entorno da RBMSC, obtendo-se nove $(n=9)$ conversas pré-entrevistas e treze $(n=13)$ entrevistas completas. Estas últimas forneceram informações sobre as relações que as pessoas têm com a RBMSC (Q 1) e sobre a presença do animal na área (Q 2). Esses 13 entrevistados possuíam as informações sobre H. maximiliani, e 92,3\% deles $(n=12)$ são antigos moradores da Fazenda Santa Cândida, uma propriedade que era abastecida por reservatórios de água que se localizam dentro da RBMSC atualmente.

Em relação às informações pessoais de cada participante das entrevistas, $81,8 \%(\mathrm{n}=18)$ eram do sexo masculino e 18,1\% ( $n=4)$ eram do sexo feminino. A idade variou entre 47 e 65 anos, sendo que 95,4\% $(n=21)$ disseram apresentar Ensino Fundamental Incompleto e apenas um informante afirmou possuir Ensino Médio Completo.

Os moradores descrevem e reconhecem $H$. maximiliani a partir de características morfológicas. Quatro dos 13 entrevistados descreveram a espécie como um animal que possui "casco meio redondo ou arredondado" e uma "cabeça comprida". Um morador local demonstrou, a partir da movimentação lateral de seu antebraço, a forma como o cágado movimenta a cabeça. Em relação à identificação do 
quelônio: 84,6\% ( $\mathrm{n}=11)$ dos moradores locais indicaram $H$. maximiliani na prancha; $30,7 \%(\mathrm{n}=4)$ indicaram Phrynops geoffroanus (Schweigger, 1812), conhecida também como cágado-de-barbicha, sendo uma espécie comum de cágado encontrada em Minas Gerais (VOGT et al., 2015b); um (7,6\%) indicou o animal Chelus fimbriatus (Schneider, 1783) (mata-matá), que ocorre na Amazônia (VOGT et al., 2015c), como o quelônio que ocorre na RBMSC.

Em relação à denominação da espécie, o nome "cágado" foi o termo popular empregado por todos os moradores locais (Q 4). Não existem trabalhos científicos nos quais populações locais nomeiam $H$. maximiliani como cágado pescoço-de-cobra, sendo este o nome caracterizado como vernacular na literatura científica (SOUZA, 2005). No caso da comunidade do entorno da RBMSC, essa denominação é desconhecida. Um morador local citou a espécie como "parente do peixe, da mesma família". Este tipo de comparação em relação a vários tipos de animais aquáticos já foi relatado em outros trabalhos (SILVA, 1989; MARQUES, 1995; ALVES et al., 2002; PEZZUTI et al., 2010; CRISTO et al., 2017).

Sobre a biologia de $H$. maximiliani, o conhecimento dos moradores entrevistados indicou que a espécie em questão é predominantemente aquática $(\mathrm{n}=10)$, sendo que três moradores informaram que a espécie habita ambientes lamacentos (Q 6). Em relação à dieta do animal, destaca-se a preferência alimentar por "insetos da água" $(\mathrm{n}=1)$, "girino" $(\mathrm{n}=3)$, "folhas e mato" $(\mathrm{n}=3)$, "lodo" $(\mathrm{n}=1)$, até "peixinhos e alevinos" ( $\mathrm{n}=5)(\mathrm{Q} 5)$, indicando onivoria. Quando perguntados sobre a reprodução da espécie, dois moradores disseram não saber e 11 responderam que H. maximiliani "botam ovos", reconhecendo que a espécie realiza a postura de ovos. Apesar de informarem acreditar na existência de alguma diferença morfológica entre machos e fêmeas (Q 7), estes moradores não relataram dimorfismo sexual na espécie.

Quanto ao uso da espécie como recurso alimentar, apenas um entrevistado mencionou já ter se alimentado do cágado: "Meu pai caçava e a gente comia, mas era só de vez em quando. Porque a gente não comia essas carnes comuns sempre. Era caro." (Q 8). Percebe-se que o uso da carne como alimento era uma estratégia para compensar a proteína animal que nem sempre era presente na alimentação da família. Segundo Cristo et al. (2017), destaca-se que em comunidades locais na Ilha de Marajó, no Pará, o cágado Kinosternon scorpioides (Linnaeus, 1766) também fazia parte na dieta de muitas famílias em momentos de escassez de proteína animal. De fato, retornos energéticos, preferências individuais, valores de mercado, abundância e facilidade de acesso são fatores que determinam o uso de animais silvestres na alimentação humana (BEGOSSI e RICHERSON, 1992; PEZZUTI et al., 2010; CRISTO et al., 2017).

Além disso, ressalta-se que o processo de urbanização altera os modos de vida de comunidades locais (GANDOLFO e HANAZAKI, 2011; TURREIRA-GARCÍA et al., 2017) e a facilitação de acesso às fontes alimentares nos mercados, como carnes bovinas, suínas e de aves, pode ter contribuído para a ausência ou perda de hábitos alimentares da comunidade local por H. maximiliani. Por fim, torna-se factível acrescentar que valores étnicos e culturais também desempenham papéis importantes no que diz respeito aos hábitos alimentares (KIMIYWE et al., 2007; NASI et al., 2011; MORCATTY e VALSECCHI, 2015).

É importante levar em consideração que práticas de caça e venda de quelônios utilizados como iguarias na culinária, além da maior afinidade pelo sabor alimentar, são mais difundidos na Amazônia brasileira, boliviana, peruana, venezuelana e colombiana, tornando essas práticas tradicionais aos indígenas e ribeirinhos que historicamente colonizaram a região norte brasileira (SOINI, 1997; FACHÍN-TERÁN, 2003; CONWAY-GÓMEZ, 2007; PEZZUTI et al., 2010; SCHAAN, 2010; ALVES et al., 2012; PEÑALOZA et al. 2013; MORCATTY e VALSECCHI, 2015). Essa realidade é distinta da encontrada na região de estudo, colonizada predominantemente por europeus com outros hábitos culturais. Essa relação entra em consonância com Redford (1982), que respalda diferenças entre hábitos de caça entre indígenas e caçadores com descendência europeia. Portanto, propõe-se que o consumo de carne pela comunidade do entorno da Reserva não seja recorrente devido a aspectos colonizatórios, culturais e ao avanço da urbanização.

Nesse mesmo contexto, a utilização do cágado como zooterápico mostrou ser desconhecida pelos moradores locais, visto que não houve relatos quanto ao uso de qualquer parte do corpo destes animais para essa finalidade. Contudo, tal uso já foi descrito na literatura para outras espécies em outras regiões do país como, por exemplo, o uso da banha sendo caracterizado como eficaz no combate ao reumatismo, asma, dor de ouvido, coceira e inchaço (COSTA NETO, 1996; 1999; ALVES et al., 2002; ALVES et al., 2011). Assim, sugere-se que, neste caso, a utilização da espécie para fins zooterápicos está ligada a práticas culturais regionais, por isso o desconhecimento deste fim na região estudada. Em relação aos 
usos alimentares, por $H$. maximiliani ser um cágado de pouco encontro na natureza (CARPENTER e FERGUSON, 1977), sugere-se que a utilização da espécie, aliada às questões histórico-culturais mencionadas anteriormente, possa estar relacionada de acordo com a sua disponibilidade no ambiente, como propõem Alves et al. $(2007 ; 2008)$ para outras espécies e regiões.

Nota-se que o conhecimento apresentado pelos moradores entrevistados acerca da identificação, classificação, biologia e uso de $H$. maximiliani foi oriundo de uma compreensão universal, atribuída similarmente por diferentes comunidades humanas a respeito das inter-relações com cágados (PEZZUTI et al., 2010; ALVES, 2012; MORCATTY et al., 2015; CRISTO et al., 2019). Esse cenário converge com a perspectiva ideacionista da relação do ser humano com a biodiversidade (HAYS, 1982). No entanto, percebe-se que a comunidade local parece não estabelecer uma ligação direta com a espécie investigada em comparação com pesquisas similares sobre etnobiologia de cágados, na região amazônica por exemplo. Tendo em vista que, nesses tipos de trabalhos, há uma maior riqueza de informações sobre conhecimento e uso de diferentes espécies para caça, alimentação, zooterapia, propriedades místico-religiosas dentre outras particularidades individuais (PEZZUTI et al., 2010; ALVES, 2012; MORCATTY et al., 2015; CRISTO et al., 2019).

Por outro lado, essa conjuntura não pode gerar uma interpretação equivocada de que a comunidade local em estudo apresenta um conhecimento inferior ou inexistente. Deve-se considerar que o conhecimento local e popular é um elemento que pode ser transmitido e aprendido entre gerações e/ou pessoas de uma comunidade, e que esses processos dependem da relevância sociocultural para determinado grupo (SOLDATI e ALBUQUERQUE, 2016; SILVA et al., 2017). Assim, é importante levar em consideração que inter-relações etnobiológicas criadas e transmitidas ao longo das gerações são moldadas por processos que tangem à sobrevivência humana (HUNN, 1982; PRADO e MURRIETA, 2015; NOVATO et al. 2020).

Nesse sentido, o processo de urbanização pode ser novamente utilizado como um fator que permite explicar um possível processo de erosão do conhecimento local sobre $H$. maximiliani. Visto que este conhecimento não se torna crucial para o cotidiano dos moradores, passando a não ter mais o valor essencial de sobrevivência e acaba se perdendo ao longo das gerações (GANDOLFO e HANAZAKI, 2011; SEYLER et al., 2019). Assim, em concordância com Turreira-García et al. (2017), defende-se que em comunidades rurais, as condições que podem afetar a transmissão do conhecimento são menores que em comunidades urbanas.

Em relação ao contato da população do entorno com a RBMSC, nove moradores disseram não possuir contato com a área e que desconhecem a presença do cágado. Contudo, afirmaram saber da existência da Reserva, mas não a frequentam, pois a entrada de pessoas não autorizadas é proibida. De Pourcq et al. (2017) consideram que as APs em geral podem exercer um papel de políticas de conservação que excluem comunidades locais e que, segundo McShane et al. (2011), abrem espaço para a formação e disseminação de situações de conflito. Nessa perspectiva, informações complementares surgiram no decorrer das entrevistas com alguns informantes. Houve relatos de seis moradores locais sobre ocupação do território da Reserva por parte de moradores dos bairros do entorno, porém essas informações se contrapuseram de acordo com o bairro em que o informante residia. Segundo os informantes, essa ocupação se dava tanto para retirada de recursos vegetais (madeira e bambu), como para construção de moradias. Novelli e Sousa (2004) e Lima (2005) já haviam registrado esse conflito de extração de recursos por parte da população local. Geraldo (2014) também relata a instalação de um conjunto habitacional pela Prefeitura ao lado da RBMSC sem considerar a zona de amortecimento necessária para a Unidade de Conservação (UC) (ver Figura 1).

Em contrapartida, no presente estudo, os tipos de contatos relatados pelos moradores locais foram: dois disseram que coletam recursos naturais na área da Reserva; dois $(n=2)$ que vão a passeio e um que vai a trabalho, enquanto oito não possuem mais contato (Q 1). Contudo, de acordo com o Sistema Nacional de Unidades de Conservação da Natureza (SNUC) (BRASIL, 2000), as Reservas Biológicas são uma tipologia de UC do grupo de proteção integral, que não permitem a presença humana, exceto para fins de pesquisa ou ações de educação ambiental. Assim, a criação da RBMSC pode ter gerado um afastamento das pessoas que frequentavam e utilizavam o local, por conta do seu caráter restritivo. Por outro lado, mesmo com esse caráter, alguns moradores continuam frequentando o local, o que indica uma resistência ou um desconhecimento das diretrizes dessa desta Lei.

Em relação à proteção da Reserva (Q 12), os treze moradores locais relataram sua importância, principalmente para preservar as nascentes de água $(n=8)$. Na pesquisa de Novelli e Sousa (2004), que também apresentaram viés etnobiológico, a maioria dos informantes não citou a preservação da Reserva 
como uma ação necessária. Já em relação ao estudo aqui discutido, todos os informantes $(\mathrm{n}=22)$ argumentaram que a Prefeitura Municipal de Juiz de Fora deveria ser a responsável pela gestão e manutenção da Reserva, visto que, segundo eles, o local encontra-se abandonado.

A partir dessas informações, sugere-se que a RBMSC foi criada por um processo onde a população local não foi inserida, dada à relação de conflito. Além disso, a ausência de cuidados com o território e a falta de fiscalização, por parte do órgão público responsável, é uma circunstância bastante comum em UC municipais no estado de Minas Gerais. De acordo com Salvio (2017), a maioria dos municípios do Estado não possui órgãos executivos de meio ambiente, e a gestão dos municípios, de modo geral, desconhece os benefícios que as UCs podem trazer para seu espaço. Sendo assim, foi possível observar que a população do entorno não está presente nos processos de proteção da biodiversidade da RBMSC, e os conflitos existentes podem prejudicar o meio natural local.

\section{CONCLUSÕES}

O conhecimento dos moradores locais sobre $H$. maximiliani, em geral, não tem relação com o uso direto da espécie, sendo este transmitido entre os antepassados da comunidade. Vale ressaltar também que o contato desta comunidade com a RBMSC ocorreu por processos individuais. Como o ambiente e cultura estão em constantes transformações, novos costumes típicos da vida urbana foram se inserindo, mas não necessariamente eliminaram a realidade anterior, vivida pelos moradores da antiga Fazenda Santa Cândida.

Aparentemente a criação Reserva não influenciou no conhecimento dos informantes quanto aos cágados, pelo fato deste animal não fazer parte do cotidiano das pessoas do entorno. Portanto, concluise que a relação dos informantes com a Reserva está mais atribuída à extração de recursos, especialmente vegetais.

Dessa forma, este trabalho constitui o primeiro contexto em que a etnobiologia foi usada para descrever esse conhecimento no ambiente urbano do entorno da RBMSC. A compreensão da relação entre comunidade do entorno e a espécie $H$. maximiliani deve ser utilizada como item fundamental nas estratégias de conservação da biodiversidade.

Assim, cabe às entidades públicas responsáveis incluírem representantes da comunidade local nos processos de proteção da RBMSC, como estratégia de minimizar os conflitos socioambientais locais. Também, sugere-se que a comunidade acadêmica atue em ações de educação ambiental a partir de projetos de pesquisa e extensão. Esses projetos podem estreitar as relações entre ciência e população e atuar como uma estratégia de repensar comportamentos humanos que podem prejudicar a manutenção dos recursos naturais.

\section{AGRADECIMENTOS}

Agradecemos ao PIBIC/UFJF/CAPES, responsáveis pelo financiamento da bolsa de Iniciação Científica deste trabalho. Aos informantes parceiros desta pesquisa, ao CNPq e aos companheiros de campo.

\section{REFERÊNCIAS}

ALBUQUERQUE, U. P.; et al. Methods and Techniques in Ethnobiology and Ethnoecology. 2. ed. Nova Iorque: Humana Press, 2019. 342p.

ALVES, A. G. C.; SOUTO, F. J. B.; LEITE, A. M. Etnoecologia dos cágados-d'água Phrynops spp. (Testudinomorpha: Chelidae) entre pescadores artesanais no açude Bodocongó, Campina grande, Paraíba, Nordeste Do Brasil. Sitientibus Série Ciências Biológicas, v. 2, n.1/2, p. 62-68, 2002.

ALVES, R. R. N. Relationships between fauna and people and the role of ethnozoology in animal conservation. Ethnobiology and Conservation, v. 1; n. 2, p. 1-69, 2012.

ALVES, R. R. N.; ALBUQUERQUE, U. P. Ethnozoology: Animals in our lives. 1. ed. Londres: Academic Press, 2018. $552 \mathrm{p}$

ALVES, R. R. N.; ROSA, I. L.; SANTANA, G. G. The role of animal-derived remedies as complementary medicine in Brazil. BioScience, v. 57, p. 949-955, 2007.

ALVES, R. R; VIEIRA, W. L. S; SANTANA, G. G. Reptiles used in tradional folk medicine: conservation implications. Biordiversity and Conservation, v. 17, n. 8, p. 2037-2049, 2008.

ALVES, R. R. N.; et al. A review on human attitudes towards reptiles in Brazil. Environ Monit Assess, v. 184, n.11, p. 6877-6901, 2011.

BAILEY, K. Methods of social research. 4 ed. Nova Iorque: The Free Press, 1994. 588 p.

BEGOSSI, A.; RICHERSON, P. J. The animal diet of families from Búzios Island (Brazil): an optimal foraging approach. Journal of Human Ecology, v. 3, p. 433-458, 1992. 
Pereira et al. Saberes populares sobre Hydromedusa maximiliani (MIKAN, 1820) (Testudines, Chelidae): uma abordagem no entorno de uma unidade de conservação urbana em Minas Gerais, Brasil. Ethnoscientia 5, 2020. DOI.: 10.22276/ethnoscientia.v5i1.312

BRASIL. Ministério do Meio Ambiente. Lei n ${ }^{\circ}$ 9.985, de 18 de julho de 2000. Institui o Sistema Nacional de Unidades de Conservação da Natureza e dá outras providências.

CARPENTER, C. C.; FERGUSON, G. W. Variation and evolution of stereotyped behavior in reptiles. In: GANS, C.; TINKLE, D. W. (Ed.). Biology of the reptilia: Ecology and Behavior. Londres: Academic Press, 1977. V. 7, p. 335-554.

CHAGAS, A. C. S.; RAPOSO-FILHO, J. R. Biologia do comportamento de Hydromedusa Maximiliani (Mikan, 1820) (Testudines: Chelidae) na Reserva Biológica Santa Cândida em Juiz de Fora - MG. Bioscience Journal, v. 15, n. 2, p. 15-23, 1999.

CONWAY-GÓMEZ, K. Effects of human settlements on abundance of Podocnemis unifilis and P. expansa turtles in Northeastern Bolivia. Chelonian Conservation and Biology, v. 6, n. 2, p. 199-205, 2007.

COSTA, H. C.; et al. New Distribution Records and Potentially Suitable Areas for the Threatened SnakeNecked Turtle Hydromedusa maximiliani (Testudines: Chelidae). Chelonian Conservation and Biology, v. 14, n. 1, p. 88-94, 2015.

COSTA-NETO, E. M. Faunistic resources used as medicines by an afro-brazilian community from Chapada Diamantina National Park, State of Bahia, Brazil. Sitientibus, n.15, p. 211-219, 1996.

COSTA-NETO, E. M. "Barata é um santo remédio": introdução à zooterapia popular no estado da Bahia. Feira de Santana: UEFS, 1999. 103 p.

CRISTO, S. S.; et al. The trade of Kinosternon scorpioides on Marajó island, Brazilian Amazon: from hunting to consumption. Herpetological Journal, v. 27, p. 361-367, 2017.

DE POURCQ, K.; et al. Understanding and Resolving Conflict Between Local Communities and Conservation Authorities in Colombia. World Development, v. 93, p. 125-135, 2017.

FACHÍN-TERÁN, A. Preservação de quelônios aquáticos com participação comunitária na Reserva de Desenvolvimento Sustentável Mamirauá, Amazonas, Brasil. In: CAMPOS-ROZO, C. ULHOA, A. (Org.). Fauna Socializada: tendencias en el manejo participativo de la fauna en América Latina. Bogota: Fundación Natura, 2003, v. 3, p. 145-176.

GANDOLFO, E. S.; HANAZAKI, N. Etnobotânica e urbanização: conhecimento e utilização de plantas de restinga pela comunidade nativa do distrito do Campeche (Florianópolis, SC). Acta Botanica Brasilica, v. 25, n. 1, p. 168-177, 2011.

GERALDO, W. M. de J. A reestruturação urbana pós-fordista de Juiz de Fora. 2014. 271 páginas. Dissertação (Mestrado em Geografia) - Universidade Federal Fluminense, Niterói.

GUIMARÃES, E.; PELLIN, A. BiodiverCidade: desafios e oportunidades na gestão de áreas protegidas urbanas. 1. ed. São Paulo: Matrix, 2015. 200p.

HAYS, T. E. Utilitarian/adaptationist explanations of folk biological classification: some cautionary notes. Journal of Ethnobiology, v. 2, p. 89-94, 1982.

HUNN, E. S. The utilitarian factor in folk biological classification. American Anthro- pologist, v. 84, p. $830-847,1982$.

ICMBIO-RAN. Centro Nacional de Pesquisa e Conservação de Répteis e Anfíbios. 2009-2011. Disponível em: <http://www4.icmbio.gov.br/ran/index.php>. Acesso em: 24 mar. 2020.

ICMBIO. Instituto Chico Mendes de Conservação da Biodiversidade. Livro Vermelho da Fauna Brasileira Ameaçada de Extinção: Volume I. 1. ed. Brasília, DF: ICMBio/MMA. 2018. 492 p.

IUCN. Red List of Threatened Species. Version 2016. Tortoise \& Freshwater Turtle Specialist Group 1996. Hydromedusa maximiliani. Disponível em: <https://www.iucnredlist.org/species/10309/97269236>. Acesso em: 23 mar. 2020.

JUIZ DE FORA. Lei n. ${ }^{\circ}$ 6910, de 31 de maio de 1986. Dispõe sobre o ordenamento do uso e ocupação do solo no Município de Juiz de Fora.

KIMIYWE, J.; WAUDO, J.; MBITHE, D.; MAUNDU, P. Utilization and medicinal value of indigenous leafy vegetables consumed in urban and peri-urban Nairobi. African Journal of Food, Agriculture, Nutrition and Development, v. 7, p. 1-15, 2007.

LIMA, A. F. B. Dieta, forrageamento, morfologia e uso de microhábitat de Enyalius perditus Jackson, 1978 (Squamata, Leiosauridae) na Reserva Biológica Municipal Santa Cândida, Juiz de Fora, Minas Gerais. 2005. 84 páginas. Dissertação (Mestrado em Comportamento e Biologia Animal) - Instituto de Ciências Biológicas, Universidade Federal de Juiz de Fora, Juiz de Fora.

MARQUES, J. G. W. Pescando pescadores: etnoecologia abrangente no baixo São Francisco alagoano. São Paulo: NUPAUB-USP, 1995. 285 p.

MENEZES, P. da. C. e. Raising the priority of urban areas in protected area systems in Brazil and beyond. In: TRZYNA, T. (Ed.). The Urban Imperative: Urban Outreach Strategies for Protect Areas Agencies. Sacramento: California Institute of Public Affairs, 2005. 168 p.

MCSHANE, T. O.; et al. Hard choices: making trade-offs between biodiversity conservation and human well-being. Biological Conservation, v. 144, p. 966-972, 2011.

MORCATTY, T. Q.; VALSECCHI, J. Social, biological, and environmental drivers of the hunting and trade of the endangered yellow-footed tortoise in the Amazon. Ecology and Society, v. 20, n. 3, p. 1-10, 2015. 
NASI, R.; TABER, A.; VAN VLIET, N. Empty forests, empty stomachs? Bushmeat and livelihoods in the Congo and Amazon Basins. International Forest Review, v. 13, p. 355-368, 2011.

NOVATO, T. S.; SOLDATI, G. T.; LOPES, J. The Agroecology Power: How the environmental representation and management of leaf-cutting ants by peasants from Assentamento Dênis Gonçalves can be transformed. Ethnobiology and Conservation, v. 9, n. 26, 2020.

NOVELLI, I. A.; et al. Alimentary habits of Hydromedusa maximiliani (Mikan, 1820) (Testudines, Chelidae) and its relation to prey availability in the environment. Herpetology Notes, v. 6, p. 503-511, 2013.

NOVELLI, I. A.; et al. First occurrence of Spirocamallanus sp. (Nematoda, Camallanoidea) in a freshwater turtle, Hydromedusa tectifera (Cope, 1869) (Testudines, Chelidae), from Brazil. Herpetology Notes, v. 7, p. 599602,2014

NOVELLI, I. A; SOUSA, B. M. Proposta Preliminar de educação ambiental para a preservação do cágado Hydromedusa maximiliani (Testudinata, Chelidae) na Reserva Biológica Municipal Santa cândida, Juiz de Fora, MG. In: XI Seminário de Iniciação Científica da UFJF. 2004.

NOVELLI, I. A; SOUSA, B. M. Análise descritiva do comportamento de corte e cópula de Hydromedusa maximiliani (Mikan, 1820) (Testudines, Chelidae) em laboratório. Revista Brasileira de Zoociências, v. 9, p. 43$50,2007$.

NOVELLI, I. A; SOUSA, B. M. Hydromedusa maximiliani (Brazilian Snake-necked turtle). Recent hatchling body mass and size. Herpetological Review, v. 39, p. 344-345, 2008.

NOVELLI, I. A; SOUSA, B. M.; DIAS, I. C. A. Hydromedusa maximiliani (Brazilian Snake-necked Turtle) algal colonization. Herpetological Review, v. 40, p. 336-336, 2009.

NOVELLI, I. A.; VIEIRA, F. M.; SOUSA, B. M. Hydromedusa maximiliani (Brazilian Snake-necked Turtle) grooming behaviour. Herpetological Review, v. 40, p. 435-436, 2009.

PEÑALOZA, C. L. O.; HERNÁNDEZ, R.; ESPÍN, L. B.; CROWDER; BARRETO, G. R. Harvest of endangered ideneck river turtles (Podocnemis spp.) in the middle Orinoco, Venezuela. Copeia, v. 2013, n. 2, p. 111-120, 2013

PRADO, H. M.; MURRIETA, R. S. S. A Etnoecologia em perspectiva: origens, interfaces e correntes atuais de um campo em ascensão. Ambiente \& Sociedade, v. 18, n. 4, 139-160, 2015.

PFJ. 2018. Prefeitura Municipal de Juiz de Fora. Sistema Municipal de Planejamento do Território SISPLAN.

<https://www.pjf.mg.gov.br/desenvolvimentodoterritorio/sistema_informacoes/areas_verdes.php>. Acesso em 23 abr. 2020.

PEZZUTI, J. C. B.; et al. Uses and taboos of turtles and tortoises along Rio Negro, Amazon Brasin. Journal of Ethnobiology, v. 30, n. 1, p. 153-168, 2010.

RAFAEL, L. M. Considerações sobre a seleção de Áreas Protegidas, o Planejamento Sistemático da Conservação e a escala de abordagem. In: XVII Simpósio Brasileiro de Geografia Física Aplicada e I Congresso Nacional de Geografia Física, p. 1214-1223. 2017.

REDFORD, K. H. The Empty Forest. BioScience, v. 42, n. 6, p. 412-422, 1992.

ROCHA, C. F. D.; et al. Répteis e sua conservação no Estado do Rio de Janeiro. In: BERGALLO, H.G.; et al. (Eds.). Estratégias e ações para a conservação da biodiversidade no Estado do Rio de Janeiro. Rio de Janeiro: Instituto Biomas, 2009. Cap 13, p. 183-191.

SALVIO, G. M. M. Áreas Naturais Protegidas e Indicadores Socioeconômicos: o desafio da conservação da natureza. 1. ed. Jundiaí: Paco Editora, 2017. 216 p.

SANDRONI, L. T.; CARNEIRO, M. J. T. "Conservação da Biodiversidade" nas Ciências Sociais Brasileiras: uma revisão sistemática de 1990 a 2010. Ambiente \& Sociedade, v. 19, n. 3, p. 21-46, 2016.

SCHAAN, D. Long-term human induced impacts on Marajó Island Landscapes, Amazon Estuary. Diversity, v.2, p. 182-206, 2010

SEYLER, B. C.; et al. Understanding knowledge threatened by declining wild orchid populations in an urbanizing China (Sichuan). Environmental Conservation, v. 46, n. 4, p. 318-325, 2019.

SHANAHAN, D. F.; et al. Toward improved public health outcomes from urban nature. American Journal of Public Health, v.105, p. 470-477, 2015.

SILVA, G. O. "Tudo que tem na terra tem no mar": a classificação dos seres vivos entre os trabalhadores da pesca em Piratininga, Rio de Janeiro. Rio de Janeiro: FUNARTE, Instituto Nacional do Folclore, 1989. 90 p.

SILVA, R. H.; et al. Human mnesic performance in a survival scenario: the application of the adaptive memory concept in ethnobiology. Ethnobiology and Conservation, v. 6, n. 9, p. 1-6, 2017.

SOANES, K.; et al. Correcting common misconceptions to inspire conservation action in urban environments. Conservation Biology, v. 33, n. 2, p. 300-306, 2019.

SOINI, P. Ecologia y manejo de quelonios acuáticos en la Amazonía peruana. In: FANG, T.; BODMER, R.; AQUINO, R.; VALQUI, M. Manejo de fauna silvestre en la Amazonía. UNAP/ Un.; Florida/UNDP, 1997. p. $167-174$

SOLDATI, G. T.; ALBUQUERQUE, U. P. Are the evolutionary implications of vertical transmission of knowledge conservative? Ethnobiology and Conservation, v.5, n. 2, p. 1-9, 2016. 
SOLIKUA, O.; SCHRAML, U. Making sense of protected area conflicts and management approaches: A review of causes, contexts and conflict management strategies. Biological Conservation, v. 222, p. 136 - 145 , 2018.

SOUZA, F. L. The Brazilian Snake-necked Turtle, Hydromedusa maximiliani. Reptilia, v. 40, p. 47 - 51, 2005.

TURREIRA-GARCÍA, N.; et al. Diversity, knowledge and use of leafy vegetables in Northern Thailand maintenance and transmission of ethnobotanical knowledge during urbanisation. Natural History Bulletin of the Siam Society, v. 62, n. 1, p. 85-105, 2017.

VOGT, R. C.; et al. 2015. Avaliação do Risco de Extinção de Hydromedusa maximiliani (Mikan, 1825) no Brasil. Processo de avaliação do risco de extinção da fauna brasileira. ICMBio. Disponível em: <http://www.icmbio.gov.br/portal/biodiversidade/fauna-brasileira/estado-de-conservacao/7403-repteishydromedusa-maximiliani-cagado-da-serra.html>. Acesso em: 25 mar. 2020.

VOGT, R. C.; et al. 2015. Avaliação do Risco de Extinção de Phrynops geoffroanus (Schweigger, 1812) no Brasil. Processo de avaliação do risco de extinção da fauna brasileira. ICMBio. Disponível em: <http://www.icmbio.gov.br/portal/biodiversidade/fauna-brasileira/estado-de-conservacao/7418-repteisphrynops-geoffroanus-cagado-de-barbicha.html>. Acesso em: 08 mai. 2020.

VOGT, R. C.; et al. 2015. Avaliação do Risco de Extinção de Chelus fimbriata (Schneider, 1783) no Brasil. Processo de avaliação do risco de extinção da fauna brasileira. ICMBio. Disponível em: <http://www.icmbio.gov.br/portal/biodiversidade/fauna-brasileira/estado-de-conservacao/7397-repteis-chelusfimbriata-mata-mata.html>. Acesso em: 08 mai. 2020.

YOUNG, J. C.; et al. The emergence of biodiversity conflicts from biodiversity impacts: characteristics and management strategies. Biodivers Conserv, v. 19, p. 3973-3990, 2010. 